\title{
COMPARISON AND OSCILLATION RESULTS FOR DELAY DIFFERENCE EQUATIONS WITH OSCILLATING COEFFICIENTS
}

\author{
WEIPING YAN and JURANG YAN
}

\author{
Department of Mathematics \\ Shanxi University \\ Taiyuan, Shanxi 030006 \\ People's Republic of China
}

(Received March 25, 1993 and in revised form November 28, 1993)

ABSTRACT. In this paper we consider the oscillation of the delay difference equation with oscillating coefficients

$$
x_{n+1}-x_{n}+\sum_{i=1}^{n} p_{i}(n) x_{n-k_{1}(n)}=0, \quad n \geqslant 0 .
$$

Some comparison and oscillation results are obtained.

KEY WORDS AND PHRASES. Oscillation, delay difference equation, oscillating coefficient

1991 AMS SUBJECT CLASSIFICATION CODE. 39A12.

\section{INTRODUCTION.}

Let $R=(-\infty, \infty)$ and $Z=\{0,1,2, \cdots\}$. Consider the delay difference equation

$$
x_{n+1}-x_{n}+\sum_{i=1}^{m} p_{i}(n) x_{n-k,(n)}=0, n \geqslant 0,
$$

and the delay difference inequality

$$
x_{n+1}-x_{n}+\sum_{i=1}^{m} p_{i}(n) x_{n-k_{i}(n)} \leqslant 0, n \geqslant 0,
$$

where

$$
\begin{array}{ll}
k_{\imath}(n) \in Z & \text { for } n \in Z, \\
p_{\imath}(n) \in R & \text { for } n \in Z,
\end{array}
$$

and there exist positive $\mathrm{k}_{1}, \mathrm{k}_{\mathrm{m}} \in Z$, such that

$$
k_{1} \geqslant k_{1}(n) \geqslant k_{2}(n) \geqslant \cdots \geqslant k_{m}(n) \geqslant k_{m}>0,
$$

and the following condition(A) is satisfied for $k_{1}$,

$$
\begin{cases}(i) \quad p_{1}(n) \in R^{+}, p_{1}(n)+p_{2}(n) \in R^{+}, \cdots, \sum_{i=1}^{m} p_{i}(n) \in R^{+}=[0, \infty) ; \\ \text { (ii) } \quad \text { For any } N \in Z, \text { there exists } N_{1} \in Z \text { such that } p_{i}(n) \in R^{+} \\ \text {for any } n \in\left[N_{1}, N_{1}+k_{1}\right], \text { where } i=1,2, \cdots, m,\end{cases}
$$

where $\left[N_{1}, N_{1}+k_{1}\right]=\left\{N_{1}, N_{1}+1, \cdots, N_{1}+k_{1}\right\}$.

Let $n_{0}-k=\inf _{n \in Z}\left\{n-k_{1}(n)\right\}$ and $n_{0} \geqslant 0$. By a solution of (1.1) (or (1.2)) we mean a sequence $\left\{x_{n}\right\}$ which is defined for $n \geqslant n_{0}-k$ and satisfies (1.1) (or (2.2)) for $n \geqslant n_{0}$. With Eq. (1.1) and with a given "initial point" $n_{0} \geqslant 0$ and "initial condition" $a_{n_{0}}-k, a_{n_{\circ}}$ 
$-\mathrm{k}+1, \cdots, \mathrm{a}_{\mathrm{n}_{\mathrm{n}}}, \mathrm{Eq} .(1.1)$ has a unique solution $\left\{\mathbf{x}_{\mathrm{n}}\right\}$ which satisfies

$$
x,=a, \text { for } j=n_{0}-k, n_{0}-k+1, \cdots, n_{0} \text {. }
$$

A solution $\left\{x_{n}\right\}$ of Eq. (1. 1$)$ is said to be oscillatory if the terms $x_{n}$ of the sequence are not eventually positive or eventually negative. Otherwise, the solution is called nonoscillatory. Eq. (1.1) is called oscillatory if every solution of the equation oscillates.

A solution $\left\{x_{n}\right\}$ of Eq. (1.1)through an initial point $n_{0}$ is said to be positive if the terms $x_{n}$ of the solution $\left\{x_{n}\right\}$ are posivive for all $n \geqslant n_{0}-k$.

Recently there has been a lot of interest in the oscillations of delay difference equations. See, for example, $[1]-[5]$ and the references cited therein. Our aim in this paper is to study the oscillation of Eq. (1.1). Some necessary and sufficient conditions and some easily verifiable sufficient conditions are established for oscillation of Eq. (1.1).

\section{MAIN RESULTS.}

Consider a sequence $\left\{\mathrm{A}_{\mathrm{n}}^{(\mathrm{r})}\right\}_{\mathrm{r}}^{\infty}=0$, which is defined as

$$
A_{n}^{(0)}=0, \quad \text { for } n \geqslant n_{0}-k_{1} \text {, }
$$

and for $r \geqslant 1$,

$$
A_{n}^{(r)}=\left\{\begin{array}{l}
0, \quad \text { for } n=n_{0}-k_{1}, n_{0}-k_{1}+1, \cdots, n_{0}-1, \\
\sum_{i=1}^{m} p_{i}(n) \prod_{j=n-k_{i}(n)}^{n-1}\left(1-A_{\jmath}^{(r-1)}\right)^{-1}, \quad \text { for } n \geqslant n_{0} .
\end{array}\right.
$$

First, we introduce the following Lemmas.

LEMMA 1. Assume that condition (A) holds for $k_{1}$ and $\left\{x_{n}\right\}$ is an eventually positive solution of (1.1). Then, $\left\{x_{n}\right\}$ must be eventually nonincerasing. And, we have

$$
x_{n+1}-x_{n}+\sum_{i=1}^{m} p_{i}(n) x_{n-k_{m}} \leqslant 0 .
$$

PROOF. By condition(A), there exists $N_{1} \geqslant n_{0}$ such that

$$
x_{n+1}-x_{n} \leqslant x_{n+1}-x_{n}+\sum_{n=1}^{m} p_{1}(n) x_{n-k_{1}(n)}=0, \quad \text { for } n \in\left[N_{1}, N_{1}+k_{1}\right] \text {, }
$$

that is, $\left\{x_{n}\right\}$ is nonincreasing on $\left[N_{1}, N_{1}+k_{1}\right]$.

We claim that $\left\{x_{n}\right\}$ is nonincreasing for $n \in\left[N_{1}+k_{1}, N_{1}+k_{1}+k_{m}\right]$.

In fact, for any $n \in\left[N_{1}+k_{1}, N_{1}+k_{1}+k_{m}\right]$, we have $n-k_{1}(n) \in\left[N_{1}, N_{1}+k_{1}\right]$. By (1.4) and nonincreasing property of $\left\{\mathrm{x}_{n}\right\}$ on $\left[\mathrm{N}_{1}, \mathrm{~N}_{1}+\mathrm{k}_{1}\right]$ we have that

$$
\begin{aligned}
& x_{n-k_{1}} \geqslant x_{n-k_{1}(n)} \geqslant \cdots \geqslant x_{n-k_{-}(n)} \geqslant x_{n-k_{-}}>0, \\
& \quad \text { for any } \mathrm{n} \in\left[\mathrm{N}_{1}+\mathrm{k}_{1}, \mathrm{~N}_{1}+\mathrm{k}_{1}+\mathrm{k}_{\mathrm{m}}\right] .
\end{aligned}
$$

So, we get that

$$
\begin{aligned}
x_{n+1}-x_{n} & =-\sum_{i=1}^{m} p_{i}(n) x_{n-k_{i}(n)} \\
& =-p_{1}(n) x_{n-k_{1}(n)}-\sum_{i=1}^{m} p_{i}(n) x_{n-k_{2}(n)}
\end{aligned}
$$




$$
\begin{aligned}
& \leqslant-p_{1}(n) x_{n-k_{1}(n)}-\sum_{-2}^{m} p_{1}(n) x_{n-k_{1}(n)} \\
& \leqslant-\left(p_{1}(n) x_{n}+p_{2}(n)\right) x_{n \cdot k_{2}(n)}-\sum_{i=3}^{m} p_{i}(n) x_{n k_{1}(n)} \\
& \leqslant \cdots \cdots \\
& \leqslant-\left(p_{1}(n)+p_{2}(n)+\cdots+p_{m}(n)\right) x_{n-k_{-}(n)} \\
& \leqslant-\left(p_{1}(n)+p_{2}(n)+\cdots+p_{m}(n)\right) x_{n-k_{m}} \leqslant 0 .
\end{aligned}
$$

Therefore, $x_{n}$ is nonincreasing on $\left[N_{1}+k_{1}, N_{1}+k_{1}+k_{m}\right]$. Similarly, we can show that $\left\{x_{n}\right\}$ is nonincreasing for all $n \geqslant N_{1}+k_{1}$ and the proof is complete.

LEMMA 2. Assume that condition (A) holds for $\mathbf{k}_{1}$ and (1.1) has a positive solution. Then there exists a sequence $\left\{\alpha_{n}\right\}_{n=n_{0}-h}$ such that the following statements are true:

(i) $\quad \alpha_{n}=\sum_{i=1}^{m} p_{i}(n) \prod_{j=n-h_{i}(n)}^{n-1}\left(1-\alpha_{1}\right)^{-1}, \quad$ for $n \geqslant n_{0}$;

(ii) $\quad \alpha_{\mathrm{n}}<1 \quad$ for $\mathrm{n}=\mathrm{n}_{0}-\mathrm{k}_{1}, \mathrm{n}_{0}-\mathrm{k}_{1}+1, \cdots, \mathrm{n}_{0}-1$ and eventually $0 \leqslant \alpha_{\mathrm{n}}<1$ for $n \geqslant N_{1}$.

PROOF. Assume that $\left\{x_{n}\right\}$ is a solution of (1.1) and $x_{n}>0$ for all $n \geqslant n_{0}-k$. Set

$$
\alpha_{n}=1-\frac{x_{n+1}}{x_{n}}, \quad \text { for } n \geqslant n_{0}-k \text {. }
$$

Then

$$
\begin{aligned}
& \frac{x_{n-k_{1}(n)}}{x_{n}}=\frac{x_{n-k_{1}(n)}}{x_{n-k_{1}(n)+1}} \cdot \frac{x_{n-k_{1}(n)+1}}{x_{n-k_{1}(n)+2}} \ldots \frac{x_{n-1}}{x_{n}} \\
& =\prod_{\jmath=n-k_{1}(n)}^{n-1}\left(1-\alpha_{\jmath}\right)^{-1}, n \geqslant n_{0} .
\end{aligned}
$$

From (1.1), we have that

$$
\frac{x_{n+1}}{x_{n}}-1+\sum_{i=1}^{m} p_{i}(n) \frac{x_{n-k(n)}}{x_{n}}=0, \quad n \geqslant n_{0} .
$$

Hence, by substituting (2.4) and (2.5)into (2.6), we get

$$
\alpha_{n}=\sum_{i=1}^{m} p_{i}(n) \prod_{\jmath=n-k_{i}(n)}^{n-1}\left(1-\alpha_{\jmath}\right)^{-1}, \quad n \geqslant n_{0},
$$

that is, (i) is satisfied. Clearly, $\alpha_{n}<1$ for $n \geqslant n_{0}-k_{1}$. By Lemma 1 , we have eventually $0 \leqslant \alpha_{n}<1$. The proof of Lemma 2 is completed.

LEMMA 3. Assume that condition (A)holds for $\mathrm{k}_{1}$ and (1.1) has a positive solution through $n_{0}$. Then the sequence (2.1) is well defined for $n \geqslant n_{0}$ and satisfies

(i) $\quad 0 \leqslant A_{n}^{(r)} \leqslant A_{n}^{(r+1)}, \quad$ for $n \geqslant n_{0}$ and $r \geqslant 0$;

(ii) $\quad \lim _{r \rightarrow \infty} A_{n}^{(r)} \underline{\underline{\text { def. }}} A_{n}<1, \quad$ for $n \geqslant n_{0}$.

PROOF. Assume that $\left\{x_{n}\right\}$ is a positive solution of (1.1) through $n_{0}$. By Lemma 1 , without loss of generality, we assume $\left\{x_{n}\right\}$ nonincreasing as $n \geqslant n_{0}-k_{1}$.

Set $\alpha_{n}=1-\frac{x_{n+1}}{x_{n}}$ for $n \geqslant n_{0}-k_{1}$. Then from(2.4), and Lemma 1 , and by a simple induction, it can be seen that 


$$
0 \leqslant A_{n}^{(r)} \leqslant A_{n}^{(r+1)} \leqslant \alpha_{n}<1, \quad r \geqslant 0 .
$$

In fact, $\mathrm{A}_{\mathrm{n}}^{(0)}=0$, and

$$
A_{n}^{(1)}=\left\{\begin{array}{l}
0, \quad \text { for } n=n_{0}-k_{1}, n_{0}-k_{1}+1, \cdots, n_{0}-1 . \\
\sum_{i=1}^{n} p_{i}(n), \quad \text { for } n \geqslant n_{0} .
\end{array}\right.
$$

So, we have $A_{n}^{(1)} \geqslant A_{n}^{(0)} \geqslant 0$. Assume that $A_{n}^{(r)} \geqslant A_{n}^{(r-1)} \geqslant 0$. Then, for $n \geqslant n_{0}$, we have

$$
\begin{aligned}
& A_{n}^{(r+1)}=\sum_{i=1}^{m} p_{i}(n) \prod_{j=n-k_{i}(n)}^{n-1}\left(1-A_{\jmath}^{(r)}\right)^{-1}, \\
& A_{n}^{(r)}=\sum_{i=1}^{m} p_{i}(n) \prod_{,=n-k_{i}(n)}^{n-1}\left(1-A_{\jmath}^{(r-1)}\right)^{-1},
\end{aligned}
$$

Hence, we get

$$
\begin{gathered}
A_{n}^{(r+1)}-A_{n}^{(r)}=\sum_{i=1}^{m} p_{i}(n) \prod_{j=n-k_{i}(n)}^{n-1}\left(1-A_{\jmath}^{(r-1)}\right)^{-1}\left[\prod_{\jmath=n-k_{,}(n)}^{n-1} \frac{\left(1-A_{\jmath}^{(r-1)}\right)}{\left(1-A_{\jmath}^{(r)}\right)}-1\right] \\
\geqslant\left(\sum_{i=1}^{m} p_{i}(n)\right) \prod_{j=n-k_{m}}^{n-1}\left(1-A_{\jmath}^{(r-1)}\right)^{-1}\left[\prod_{j=n-k_{m}}^{n-1} \frac{\left(1-A_{\jmath}^{(r-1)}\right)}{\left(1-A_{\jmath}^{(r)}\right)}-1\right] \geqslant 0,
\end{gathered}
$$

so, we know that $0 \leqslant A_{n}^{(r)} \leqslant A_{n}^{(r+1)}$ for all $r \geqslant 0$.

By (2.7), we use the induction to get

$$
A_{n}^{(r)} \leqslant \alpha_{n}<1, \quad \text { for any } r \geqslant 0, n \geqslant n_{0}-k_{1},
$$

which implies that (2.8)holds. Hence it is easy to get that

$$
\lim _{r \rightarrow \infty} A_{n}^{(r)}=A_{n}<1, n \geqslant n_{0},
$$

and the proof is complete.

The next result is a generalization of Theorem 1 in [5].

THEOREM 1. Assume that (1.3), (1.4) and condition(A)hold for $\mathbf{k}_{1}$. Then the following statements are equivalent:

(a) Eq. (1.1)has a positive solution through the initial point $n_{0} \geqslant 0$;

(b) The inequality (1.2) has an eventually positive solution;

(c) The sequence $\left\{A_{n}^{(r)}\right\}_{r=0}^{\infty}$ which is well defined by (2.1)converges to a limit $A_{n}$ with $0 \leqslant A_{n}<1$ for each $n \geqslant n_{0}>0$.

PROOF. $(a) \Rightarrow(b)$. This is obvious.

(b) $\Rightarrow(c)$. Assume that $\mathbf{x}_{n}>0$ for $n \geqslant n_{0}-k$ which is a solution of (1.2). Set

$$
\tilde{\alpha}_{n}=1-\frac{x_{n+1}}{x_{n}}, n \geqslant n_{0}-k \text {. }
$$

Then,

$$
\frac{x_{n-k_{1}(n)}}{x_{n}}=\prod_{\jmath=n-k_{1}(n)}^{n-1}\left(1-\tilde{\alpha}_{\jmath}\right)^{-1}, \quad n \geqslant n_{0} .
$$

Thus from (1.2), it follows that

$$
\sum_{i=1}^{m} p_{i}(n) \prod_{j=n-k_{i}(n)}^{n-1}\left(1-\tilde{\alpha}_{\jmath}\right)^{-1} \leqslant \tilde{\alpha}_{n}, \quad n \geqslant n_{0} .
$$

By (2.1) and a simple induction which is the same as that of Lemma 3, we hvae that 


$$
0 \leqslant A_{n}^{(r)} \leqslant A_{n}^{(r+1)} \leqslant \tilde{\alpha}_{n}<1 \text { for } r \geqslant 0 \text { and } n \geqslant n_{0} \text {, }
$$

which implies that the sequence $\left\{\mathrm{A}_{n}^{(r)}\right\}$ converges 10 finite limit $\mathrm{A}_{n}$ with $0 \leqslant \mathrm{~A}_{n}<1$ for each fixed $n \geqslant n_{0}$.

(c) $\Rightarrow$ (a). It is similar to that of Theorem 1 in [5].

The proof of Theorem 1 is complete.

COROLLARY 1. Assume that (1.3), (1.4) and condition (A) hold for $\mathrm{k}_{1}$. Then the following statements are equivalent:

(a) Eq. (1.1) is oscillating;

(b) Inequality (1.2) has no eventually positive solution.

Assume that $P_{n} \in R^{+}, n \in Z$, and $k \in Z$. The following theorem of oscillation was obtained in [3].

THEOREM A. Consider the delay difference equation

If

$$
A_{n+1}-A_{n}+P_{n} A_{n-k}=0, \quad n=0,1,2, \cdots \text {. }
$$

$$
\operatorname{limin}_{n \rightarrow \infty}\left[\frac{1}{k} \sum_{i=n-k}^{n-1} P_{\imath}\right]>\frac{k^{k}}{(k+1)^{k+1}},
$$

then all solutions of $(*)$ are oscillatory.

In [5], the following conclusion was obtained.

THEOREM $\mathrm{B}$. Consider the following inequality

$$
A_{n+1}-A_{n}+P_{n} A_{n-k} \leqslant 0 \text {. }
$$

The following conclusions are equivalent :

(i) $(*)$ is oscillatory;

(ii) $(* *)$ has no eventually positive solution.

We can obtain the following theorem.

THEOREM 2. Assume that (1.3), (1.4) and condition (A)hold for $k_{1}$, and the equation

$$
x_{n+1}-x_{n}+\sum_{i=1}^{m} p_{i}(n) x_{n-k_{m}}=0
$$

is oscillatory, then (1.1) must be oscillatory.

PROOF. Let $\left\{x_{n}\right\}$ be a nonoscillatory solution of (1.1). As the opposite of a solution of (1.1) is still a solution of (1.1), we can assume that $x_{n}>0$ for $n \geqslant n_{0}$. By Lemma 1 , we have

$$
x_{n+1}-x_{n}+\sum_{i=1}^{m} p_{i}(n) x_{n-k_{m}} \leqslant 0,
$$

that is, inequality (2.12) has a positive solution. On the other hand, by Theorem B we know that (2.12) has no eventually positive solution. This is a contradiction. So, (1.1)must be oscillatory. The proof of Theorem 2 is complete.

COROLLARY 2. Assume that (1.1), (1.4), condition (A) hold for $\mathrm{k}_{1}$, and

$$
\liminf _{n \rightarrow \infty} \frac{1}{k_{m_{l}}} \sum_{n-k_{-}}^{n-1} \sum_{j=1}^{m} p,(i)>\frac{k_{m}^{k_{m}}}{\left(k_{m}+1\right)^{k_{-}+1}} .
$$

Then (1.1) is oscillatory. 
PROOF. By Theorem 2 and Theorem A, we obtain the conclusion.

COROLLARY 3. Assume that (1.3), (1.4), condition (A)hold for $\mathbf{k}_{1}$, and

$$
\sum_{i=1}^{m} p_{i}(n) \geqslant P_{n} \quad \text { for } n \text { sufficiently large. }
$$

Then, if $(*)$ is oscillatory, (1.1) must be oscillatory.

\section{REFERENCES}

1. ERBER,L. H. and ZHANG, B. G. ,Oscillation of discrete analogues of delay equation, Diff Int. Equations 2 (1989), 300-309.

2. LADAS, G., Explicit conditions for the oscillation of difference equations, J. Math. Anal. Appl. 153 (1990),276-287.

3. LADAS, G. PHILOS, Ch. G and SFICAS, Y. G. , Sharp conditions for the oscillation of delay difference equations, J. Appl. Math. Simulation 2(1989), 101-112.

4. PHILOS, Ch. G. , Oscillations in a nonautonomous delay logistic difference equation, Proceedings of the Edinbargh Mathematical Society 35 (1992), 121-131.

5. YAN, JURANG and QIAN, CHUANXI, Oscillation and comparison results for delay difference equations, J. Math. Anal. Appl. 165 (1992), 346-360. 


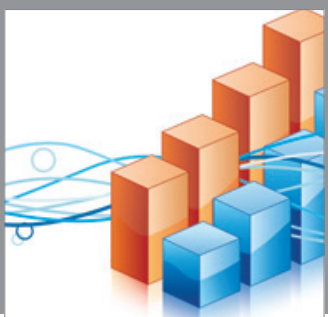

Advances in

Operations Research

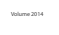

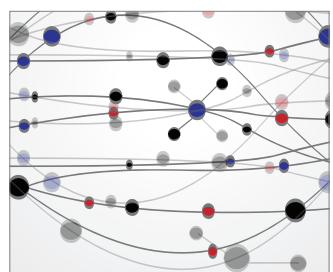

\section{The Scientific} World Journal
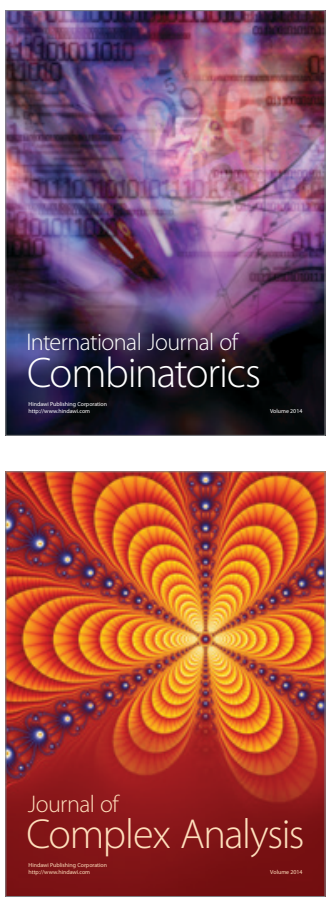

International Journal of

Mathematics and

Mathematical

Sciences
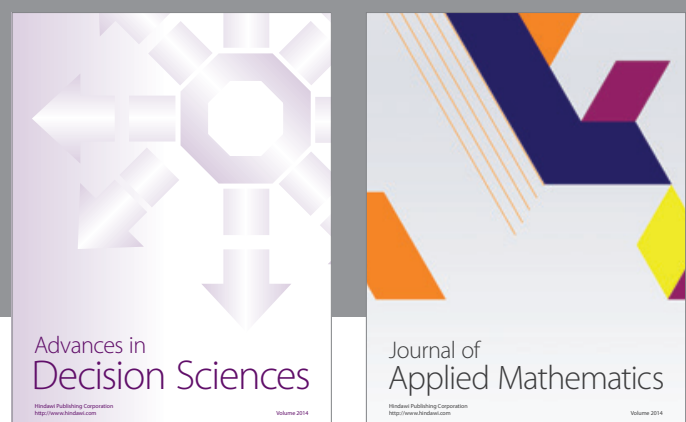

Journal of

Applied Mathematics
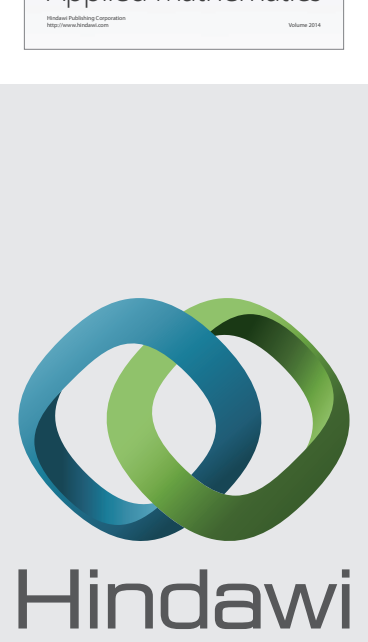

Submit your manuscripts at http://www.hindawi.com
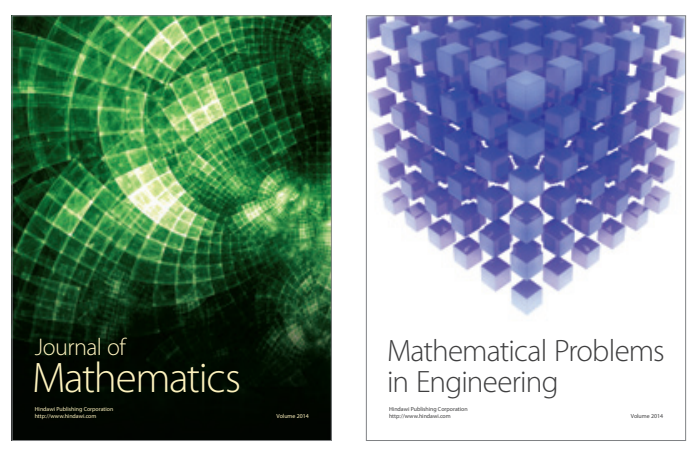

Mathematical Problems in Engineering
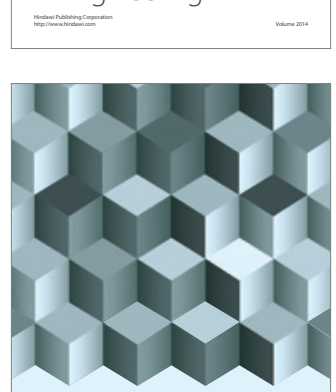

Journal of

Function Spaces
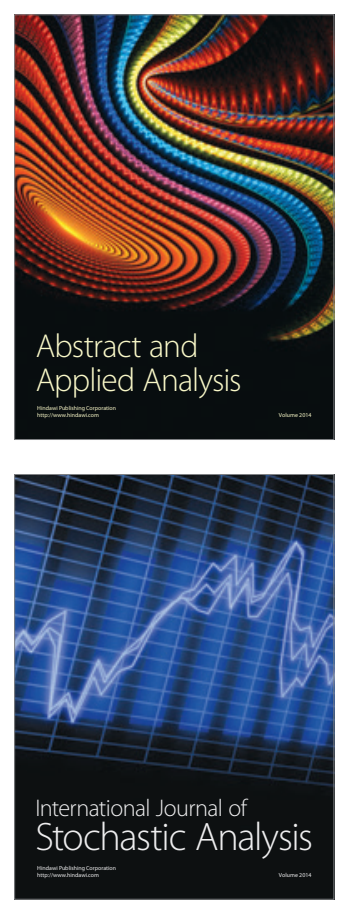

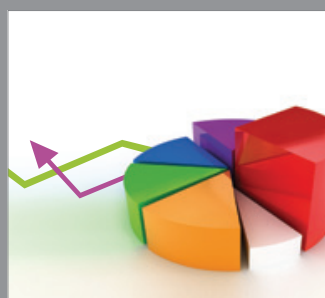

ournal of

Probability and Statistics

Promensencen
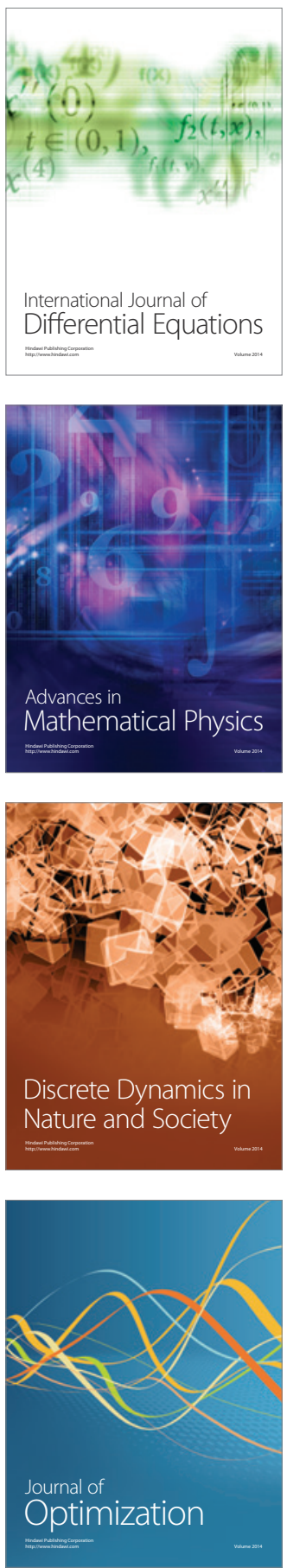